complicated by the presence of a number of thermal sources at this longitude. Furthermore, the north polar spur intersects the galactic plane at this point, so that one has to be very cautious in interpreting the results as a $3-\mathrm{kpc}$ ring of thermal material.

Westerhout: With regard to Dr. Large's remark that the "spur" might influence the bump in the galactic radiation near $l^{\prime \prime}=30^{\circ}$, it must be said that this spur clearly has a nonthermal spectrum. Also, one cannot really connect it with the main body of the Milky Way. I have the feeling that it is a completely separate phenomenon.

Mathewson: Up till now, radiative excitation has been thought responsible for the ionization of the hydrogen in this strong central thermal component. Collisional excitation should also be considered, the energy for which may be supplied by the gas which $\mathrm{H}$-line observers find to be flowing rapidly outwards from the galactic centre. Electron temperatures in excess of $10^{4}{ }^{\circ} \mathrm{K}$ could then be obtained.

Kerr: It has been suggested by two speakers that the ring of ionized hydrogen at $3 \cdot 5 \mathrm{kpc}$ lies just outside the so-called 3-kpc arm of neutral hydrogen. The latter arm can in fact be followed to longitude $334^{\circ}$. If we are seeing the arm tangentially at this longitude, the corresponding distance from the galactic centre is $3.5 \mathrm{kpc}$.

Bolton: There is no real evidence that the so-called $3 \cdot 5 \cdot \mathrm{kpc}$ ring is a complete ring. It certainly extends from the tangential points at $\pm 30^{\circ}$ longitude from the centre and on the nearby side of the centre. Very high resolution radio observations may confirm its completeness on the other side of the centre but the evidence at present only supports an arc of 120 to $180^{\circ}$.

Westerhout: Our assumption of a ring is only based on the observation that the steps at both sides of the centre occur at exactly the same distance from the centre. Indeed, one can assume that we are dealing with a part of a ring, or even with an accidental occurrence of density maxima. But I feel the symmetry argument rather strongly favours a ring or large arc structure.

\title{
29. SUPERASSOCIATIONS IN DISTANT GALAXIES
}

\section{A. Ambartsumian \\ Byurakan Observatory}

It is well known that the Large Magellanic Cloud contains in addition to a considerable number of ordinary $\mathrm{O}$-associations a certain number of large objects which, however, are similar in nature to the associations. These objects were named "constellations" by Shapley. But the large complex 30 Doradus surpasses notably all of these objects both in diameter and in absolute brightness. The latter is of the order of $-15^{\mathrm{m}} .0$ while its diameter is of the order of $600 \mathrm{pc}$. If we take the average absolute brightness of associations in our Galaxy as equal to $-10^{\mathrm{m}} .0$ then it turns out that 30 Doradus is 100 times more luminous than the ordinary associations. The photographic images of more distant galaxies reveal that sometimes complexes occur in them of the same order of luminosity and dimensions as 30 Doradus. Therefore it seems to us useful to regard these complexes as a special class of objects and call them superassociations.

The frequency of occurrence of superassociations within the galaxies is being investigated at the Byurakan Observatory. On plates taken by means of the 21 -inch Schmidt reflector the superassociations are almost star-like if the distance of the corresponding galaxy is over 15 million pc. When exposures are of shorter duration (a few seconds) the general background of the corresponding galaxy does not hinder photometric evaluations, and the images of superassociations can be compared with 
the focal images of the surrounding stars or of those located in standard areas. In determining the stellar magnitude in this way the error for closer galaxies may however attain $0^{\mathrm{m}} 5$.

We quote here only the preliminary results of the review based in all on the study of 55 galaxies, mostly belonging to the Sc type. These galaxies (with the exception of two) have been selected from the Shapley-Ames catalogue at random, if we disregard the fact that Sc galaxies were preferably observed while $\mathrm{E}$ type galaxies have not been observed at all.

In Table 1 the NGC numbers of 14 galaxies are listed, in which superassociations have been found. Concentrations exceeding $-13^{\mathrm{m}} 5$ in absolute magnitude have been considered as superassociations. Naturally we could not have regarded as superassociations the long sections of the bright parts of the spiral arms.

TABLE 1

THE GALAXIES WITH SUPERASSOCIATIONS

\begin{tabular}{|c|c|c|c|c|c|}
\hline NGC & Type & $N_{\mathrm{sa}}$ & $\bar{M}_{\mathrm{sa}}$ & $M_{\mathrm{g}}$ & $M_{\mathrm{n}}$ \\
\hline 1087 & $\mathrm{Sc}$ & 8 & $-14 \cdot 9$ & $-20 \cdot 6$ & $-15 \cdot 9$ \\
\hline 1961 & $\mathrm{Sb}$ & 3 & $-15 \cdot 8$ & $-21 \cdot 5$ & $-17 \cdot 1$ \\
\hline 2276 & $\mathrm{Sc}$ & 4 & $-15 \cdot 2$ & $-20 \cdot 7$ & $-15 \cdot 9$ \\
\hline 3991 & Haro & 2 & $-17 \cdot 2$ & - & no \\
\hline 3995 & $\mathrm{Sc}$ & 4 & $-14 \cdot 8$ & $-20 \cdot 3$ & $-17 \cdot 0$ \\
\hline 4303 & $\mathrm{Sc}$ & 4 & $-14 \cdot 6$ & $-21 \cdot 6$ & $-17 \cdot 6$ \\
\hline 4496 & $\mathrm{SBc}$ & 1 & $-14 \cdot 9$ & $-19 \cdot 8$ & no \\
\hline 4559 & Sc & 2 & $-13 \cdot 6$ & $-19 \cdot 6$ & $-14 \cdot 1$ \\
\hline 5676 & $\mathrm{Sc}$ & 2 & $-15 \cdot 5$ & $-20 \cdot 8$ & $-16 \cdot 0$ \\
\hline 5678 & $\mathrm{Sc}$ & 4 & $-17 \cdot 5$ & $-20 \cdot 5$ & $-15 \cdot 8$ \\
\hline 6217 & $\mathrm{Sc}$ & 4 & $-14 \cdot 5$ & $-19 \cdot 8$ & $-16 \cdot 6$ \\
\hline 6412 & $\mathrm{Sc}$ & 1 & $-15 \cdot 5$ & $-19 \cdot 4$ & $-15 \cdot 8$ \\
\hline 6643 & $\mathrm{Sb}$ & 3 & $-14 \cdot 8$ & $-20 \cdot 0$ & $-15 \cdot 4$ \\
\hline 7448 & $\mathrm{Sc}$ & 3 & $-15 \cdot 0$ & $-20 \cdot 8$ & $-16 \cdot 2$ \\
\hline
\end{tabular}

The second column of the table reproduces the galactic types; the third column, the number of superassociations in each galaxy; the fourth column, the mean absolute magnitudes of the superassociations; the fifth, the absolute magnitudes of the galaxies (Sandage's scale of distances is assumed); and the sixth, for comparison, the absolute magnitude of the nucleus of the galaxy as determined from the same plates. The data of this table make it clear that the superassociations are met with particularly often in supergiant galaxies with an absolute magnitude of $-20 \mathrm{~m} 5$ and above. Of the observed 35 galaxies of the Sc type eight, containing superassociations, have an average absolute magnitude of $-20^{\mathrm{m}} \cdot 6$ whereas the absolute magnitude of Sc galaxies without superassociations is equal to $-18 \mathrm{~m}$. As for the Sb galaxies, the number of the observed objects is, in this case, small. It is therefore hard to speak of any existing difference. However, there is no doubt that $\mathrm{Sb}$ galaxies, containing superassociations, constitute at the same time systems of high luminosity. 
In Table 2 the numbers $(N)$ of all the observed galaxies of the Sc type are quoted for three different intervals of absolute magnitude, together with $N_{\text {sa }}$, the numbers of galaxies containing superassociations for the same intervals.

The last column deals with the average numbers of superassociations per galaxy of the given class of luminosity. This table shows more convincingly that the superassociations are encountered almost exclusively in supergiant galaxies.

Apparently the picture is somewhat different in irregular galaxies. We have not as yet treated the problem in detail, but the NGC 275 Haro-type galaxy, involving at least five superassociations and of an absolute magnitude $-19^{\mathrm{m}} \cdot 0$, testifies to the fact that in irregular galaxies the case is different. This is also attested by the example of the Large Magellanic Cloud.

TABLE 2

FREQUENCY OF SUPERASSOCIATIONS IN Sc GALAXIES

\begin{tabular}{r|r|c|c}
\hline Interval of $M$ & $N$ & $N_{\mathrm{sa}}$ & $\nu^{*}$ \\
\hline$M>-20 \cdot 0$ & & & \\
$-20 \cdot 5<M<-20 \cdot 0$ & 21 & 3 & $0 \cdot 3$ \\
$M<-20 \cdot 5$ & 6 & 1 & $0 \cdot 7$ \\
& 8 & 6 & $3 \cdot 1$ \\
\hline
\end{tabular}

* See text.

As became evident from the work of Shapley and Paraskevopoulos (1937), most of the luminosity of the 30 Doradus complex is contained in the nebula. But 30 Doradus contains also hundreds of blue supergiant stars with the richest cluster of supergiants located in the centre of this complex. It should be pointed out, at the same time, that the lifetime of the superassociations as a whole must considerably exceed the mean lifetime of ordinary associations. It simply follows from the fact that the lower limit of the duration of life of any such complex must be the value $D / v$, where $D$ is the diameter of the complex while $v$ indicates the mean relative velocity traced in it. Making $D=600 \mathrm{pc}$ and $v=10 \mathrm{~km} / \mathrm{sec}$ we obtain for the lifetime a lower limit of $6 \times 10^{7}$ years. This is nearly one order of magnitude more than the age of ordinary $\mathrm{O}$-associations and that of hot supergiants. It must therefore be assumed that the blue supergiants observed in the superassociations represent only one of the numerous generations of these objects. Many thousands of supergiants appear, presumably, during the lifetime of the superassociation, which thereupon turn into other stars. If we take into account the fact that the T-Tauri type stars usually originate in similar complexes and in far greater numbers, then we must believe that hundreds of thousands of stars come into being there.

In 1939 in the book "Theoretical Astrophysics" (Ambartsumian 1939) we made the initial estimation of the mass of the nebula 30 Doradus at $2 \times 10^{6} M \odot$, while in the Observatory at Mount Stromlo Johnson (1959) re-estimated, four years ago, the gaseous mass of this nebula. It turned out to be equal to $5 \times 10^{6} M \odot$. Consequently, it is no wonder that hundreds of thousands of stars are likely to be genetically bound with this nebula. 
In conclusion, I take the liberty of making one more remark of a cosmogonic nature.

If one realizes that superassociations are formed out of gaseous masses distributed within the given galaxy, one must believe that in galaxies of a great total mass the tidal forces should hinder the formation of such large complexes. On the contrary, in galaxies of a small mass no such hindrance will exist. For this reason we shall not expect to meet superassociations in supergiant galaxies. In fact, we note the reverse picture. This apparently refutes the assumption as to the possibility of the formation of superassociations from initial matter diffused all over the galaxy.

This paper is a preliminary report on the work being continued at Byurakan together with R. Shahbazian, S. Iskudazian, and K. Sahakian.

\section{References}

Ambartsumian, V. A. (1939).-—"Theoretical Astrophysics." (Moscow, Leningrad.)

Johnson, H. M. (1959).-P.A.S.P. 71 : 425-34.

Shapley, H., and Paraskevopoulos, J. S. (1937).-Ap. J. 86: 340-2.

\section{Discussion}

Arp: Your table showed superassociations going as bright as $M_{\mathrm{pg}}=-17^{\mathrm{m}} \cdot 5$. What would you consider the difference between a superassociation such as this and a galaxy such as the Small Magellanic Cloud?

Ambartsumian: We must consider the colour of the association, and make some sort of a limit to their absolute magnitudes. Some large complexes, such as one in M51, should probably be considered as satellite galaxies.

Oort: Are these associations always associated with spiral arms?

Ambartsumian: Some of them are situated in the spiral arms, others between the arms. There is a case in the Ursa Major cluster where the superassociation is situated on the end of the arm, a little like M51, but this case is not given in the table in the paper.

$B o k$ : Would it not be highly desirable to define superassociations as very blue in $(U-B)$ and in $(B-V)$, so that confusion with objects like the Small Magellanic Cloud might be avoided?

Ambartsumian: Yes.

Haro: Can you consider some very ultraviolet nuclei of galaxies as superassociations?

Ambartsumian: Perhaps there is something in common between the superassociations and these nuclei.

Aller: The brightest association in M33 is NGC 604, which closely resembles 30 Doradus. Do you consider this a superassociation?

Ambartsumian: I consider NGC 604 to lie just below the lower limit of what we define as a superassociation. Cloud?

Tifft: How many superassociations would you say are present in the Large Magellanic

Bok: Only one, 30 Doradus, since the rest are only slightly more luminous than normal associations.

Buscombe: Do any of the galaxies showing superassociations have a record of supernova explosions or radio sources?

Ambartsumain: I don't know.

Blaauw: With reference to the suggestion that stars (associations) may originate from super. dense bodies, I have been rather puzzled by the following aspect of it. The suggestion implies that the superdense bodies are in the plane of the galaxies considered, because that is where we find 
the associations and superassociations. How did these bodies ever get confined to this plane? If we imagine stars being formed from the interstellar medium, the location of associations in the plane is obvious, and the restriction of gas to the plane is understood as the result of the collapse of the primordial cloud. However, we know no mechanism which would have brought the (primordial) superdense bodies into the plane; for then we would rather expect to find a distribution like that of the halo stars. What do you think about this?

Ambartsumian: Of course we don't know the forces which can bring the superdense prestellar formations into the galactic plane, but it is possible that some mechanism exists.

Feast: It may be of interest to remark that quite apart from size there may be other differences between superassociations like 30 Doradus and normal associations. For instance, 30 Doradus seems to contain a much higher proportion of WR stars than the associations so far studied in the Galaxy.

de Vaucouleurs: You have indicated that 30 Doradus is a superassociation, but that NGC 604 does not qualify. Could you describe more precisely what the operational definition of superassociation is?

Ambartsumian: We define the superassociations by the absolute brightness ( $M$ brighter than $-13 \cdot 5$ ) and colours. It seems that NGC 604 falls somewhat on the borderline between normal and superassociations.

\title{
30. LARGE HI CLOUDS IN THE GALAXY
}

\author{
R. X. McGeE
}

CSIRO Radiophysics Laboratory, Sydney

\section{Introduction}

van de Hulst (1958), in giving representative sizes of the structural details of the interstellar medium, lists the dimensions of a typical cloud, seen in $21-\mathrm{cm}$ emission, as $20-70$ pc but warns that we should not be surprised to find different "sizes of interstellar clouds" determined from different sets of data.

I wish to introduce two classes of cloud or complex of neutral hydrogen of much larger dimensions than the "typical cloud":

(a) The first type, which has been observed close to the Sun, is an association presumably composed of "typical clouds" each with similar density characteristics and velocity dispersion. In general, a "typical cloud" is hard to distinguish as an individual component.

(b) The second type, observed at greater distances, is a complex several times larger. (Beyond 3 or $4 \mathrm{kpc}$ from the Sun the aerial resolution does not permit such detail as the first type to be delineated.) The clouds occur in succession along the galactic spiral arms and those mentioned here form arms at distances from the galactic centre, $R>R_{0}$ (distance Sun to centre).

The discussion is based on observations made with a low-resolution paraboloid2.2 between half-power points- and the 48-channel $\mathrm{H}$-line receiver. The bandwidth was $38 \mathrm{kc} / \mathrm{s}(\sim 8 \mathrm{~km} / \mathrm{sec}$ in radial velocity) with channel spacing at $33.2 \mathrm{kc} / \mathrm{s}$ $(\equiv 7.0 \mathrm{~km} / \mathrm{sec})$. Profiles were recorded at $2 \mathrm{~min}$ intervals in time at spacings of $1^{\circ}$ in declination between +42 and $-90^{\circ}$. The equipment is described by McGee and Murray (1963). 